\title{
Intracardiac echocardiography for transseptal puncture. A guide for cardiac electrophysiologists
}

\author{
Radu Rosu1, Gabriel Cismaru1, Lucian Muresan², Mihai Puiu1, Gabriel Gusetu', Sabina \\ Istratoaie $^{1}$, Dana Pop ${ }^{1}$, Dumitru Zdrenghea ${ }^{1}$
}

${ }^{1} 5^{\text {th }}$ Department of Internal Medicine, Cardiology-Rehabilitation, "Iuliu Hatieganu" University of Medicine and Pharmacy Cluj-Napoca, Romania, 2’"Emile Muller" Hospital, Cardiology Department, Mulhouse, France

\section{Abstract}

The key to a successful catheterization of the left heart chambers is the safe transseptal puncture. Intracardiac echocardiography (ICE) is an attractive tool used in cardiac catheterization and electrophysiology labs to provide detailed images that can facilitate transseptal puncture. ICE permits a direct visualization of the endocardium and precisely locates the needle and the sheath against the interatrial septum. Two different ICE catheters are available: a phased array and a mechanical transducer, both being currently used in clinical practice. This paper describes the technique used for guiding transseptal puncture with ICE. Due to its advantages, ICE has currently become an important tool used to maximize the safety of the transseptal puncture and increase efficacy of interventional cardiology procedures.

Keywords: transseptal puncture; intracardiac echocardiography; left atrium; interatrial septum; tenting

\section{Introduction}

In the catheterization laboratory transseptal puncture (fig 1) is mostly used for radiofrequency ablation of atrial fibrillation [1,2], left accessory pathway ablation [3], balloon mitral valvuloplasty [4], closure of the left atrial appendage [5], atrial septal defect and ventricular septal defect closure [6], patent foramen ovale (PFO) closure [7], aortic valvuloplasty or transaortic valve replacement (TAVR) [8] and pulmonary valvuloplasty [9]. The key to a successful catheterization of the left heart chambers is a safe transseptal puncture.

Intracardiac echocardiography (ICE), first described in 1981, is an attractive tool in cardiac catheterization

Received 07.12.2018 Accepted 07.02.2019

Med Ultrason

2019, Vol. 21, No 2, 183-190

Corresponding author: Gabriel Cismaru, MD

Department of Cardiology,

Rehabilitation Hospital, 46-50 Viilor Street

400347 Cluj-Napoca, Romania

Phone: (40)721926230

Fax: (40)264453131

E-mail: gabi_cismaru@yahoo.com

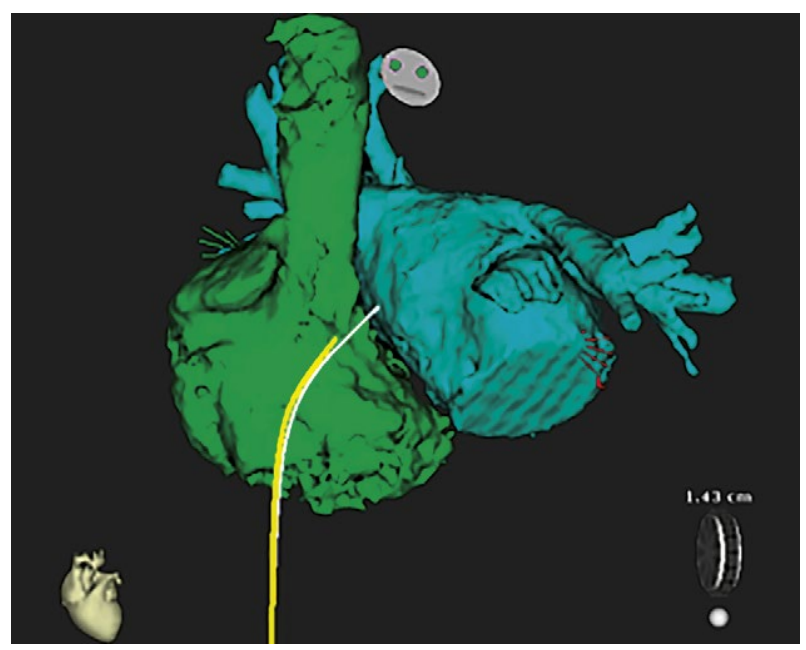

Fig 1. Computed tomography reconstruction of the left and right atrium. Fosa ovalis is the "real" interatrial septum between the right atrium and the left atrium (red circle). Around the fosa lies adipose tissue with small arterial branches, therefore the transseptal puncture should not be performed in the muscular part of the septum as it may complicate with pericardial effusion. The white arrow shows the direction of the catheter from the inferior vena cava towards the fosa ovalis during transseptal puncture. 
and electrophysiology labs to provide detailed real-time near-field and far-field images that can facilitate transseptal puncture. Images can be stored using DICOM standards and used for continuing education and medical research [10].

ICE permits direct visualization of the endocardium and precisely locates the transseptal needle and the sheath in contact with the interatrial septum. ICE should be the preferred option for guiding a safe transseptal access in cases of a modified interatrial septum anatomy: thickened septum, floppy septum, interatrial septal aneurysm, lipomatous hypertrophy, previous suture of the septum after cardiac surgery, device closure of an atrial septal defect (ASD) [11]. The technique can be used also in cases with abnormal right atrial structures that interfere with the introduction of the needle, sheath and dilator into the right atrium, such as a prominent Eustachian ridge or a Chiari network [12-14]. Transesophageal echocardiography (TEE) is an alternative to ICE in these cases. Even though TEE can provide more views than ICE, ICE reduces the patient's discomfort, the probe being introduced through the femoral vein under local anaesthesia, thus avoiding general anaesthesia and its associated risk of aspiration pneumonia [15]. Other situations where ICE guidance is desirable are lead implantation in pregnant women, extraction of cardiac devices, pacemaker related endocarditis, retrograde double balloon valvuloplasty and endomyocardial biopsy [16].

In this paper we will review in a stepwise manner the technique used for ICE-guided transseptal puncture. This technique allows a safe crossing of the interatrial septum with minimal radiation exposure and without the need of general anaesthesia.

\section{Currently available ICE systems}

For visualization of the cardiac chambers, 2 different ICE catheters are available (fig 2). The first one is the phased-array ultrasound catheter, which consists of a 64-element transducer (ViewFlex from Saint Jude or AcuNav from Siemens-Biosense Webster) that uses multiple frequencies of 5-10 MHz, providing $90^{\circ}$ sector images; it has modifiable depth control, allowing pulsed color and continuous Doppler imaging. The transducer is mounted on a $8 \mathrm{Fr}$ four-way steerable catheter (see movie 1 on the journal site), introduced through the femoral vein inside the right atrium; it permits a penetration depth ranging from $2 \mathrm{~mm}$ to $12 \mathrm{~cm}$. The second catheter (Ultra ICE from Boston Scientific) has a $9 \mathrm{MHz}$ single element mechanical transducer that is mounted on an 8 Fr nonsteerable catheter, permitting 1800 rotations per minute. This transducer allows a $360^{\circ}$ cross-sectional image in the radial plane [17].

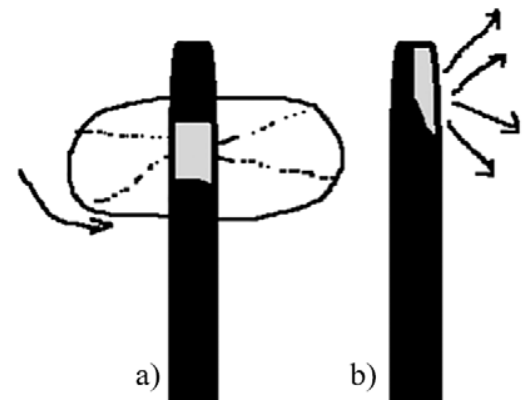

Fig 2. ICE probe. Schematic drawing of the 2 transducers types used for intracardiac echocardiography: rotational probe permits $180^{\circ}$ image (a) and phased array probe permits a $90^{\circ}$ sectorial image (b).

\section{Utility of ICE to guide the transseptal puncture}

In the center of the muscular interatrial septum lies a thin fibrous structure called the „fossa ovalis”. This structure is derived from the septum primum and septum secundum and is the true septal wall between the left atrium and the right atrium [18]. Transseptal puncture should be performed at this level to avoid any pericardial effusion. Traditionally, the localization of fossa ovalis has been done by fluoroscopy. More recently, TEE and ICE have been used to guide the transseptal puncture.

Fluoroscopy provides limited anatomical information for the right and left anatomical structures. The fossa ovalis can be located fluoroscopically in the following way: the plane of the mitral valve and the left atrium can be identified by inserting an electrophysiology catheter inserted in the coronary sinus; the position of the aortic valve can be located by placing an electrophysiology catheter at the level of His bundle or, alternatively, by placing a coronary angiography catheter in the aortic bulb, close to the aortic valve. For safety reasons, the fluoroscopic method requires 2 catheters: one in the coronary sinus and one at the level of the His or inside the aorta; a long transseptal sheath, a dilator and a Brockenbrough transseptal needle are also needed. The transseptal sheath together with the dilator are inserted into the right femoral vein and then advanced on a supporting guidewire at the level of the superior vena cava (SVC). The guidewire is then removed and the transseptal needle is inserted inside the dilator. The ensemble is then carefully retracted from the SVC into the right atrium. When the transseptal sheath is sufficiently descended and it reaches the foramen ovale, a sudden discrete movement towards the left atrium can be observed. This ,jump" is a sign that allows the operator to know that the sheath is at the level of the foramen ovale [11]. 


\section{ICE imaging to guide to transseptal puncture}

The standard approach uses a long transseptal sheath and a dilator that cover a Brockenbrough needle which are placed against the fosa ovalis. The system is introduced through the right or left femoral vein through an appropriate sized sheath $(8$ or $10 \mathrm{~F})$ until it reaches the SVC [11]. The probe is advanced with a gentle anterior tip deflection to avoid entering venous branches on the way to the right atrium. By pulling back the probe from the SVC to the inferior vena cava (IVC), important structures of the right atrium can be identified: the orifice of the SVC, the right atrial appendage, the crista terminalis, the interatrial septum, the orifice of the coronary sinus, the tricuspid valve, the orifice of the IVC. Other structures, in close proximity to the right atrium can be visualized: the pulmonary artery, the ascending aorta with the aortic root, the right ventricle, the left ventricle and the cardiac valves. Another approach is to introduce the ICE catheter into the right atrium via the right subclavian or internal jugular vein (fig 3).

\section{STEP 1: „Home view”}

In this view the transducer is located in the mid-right atrium, aligned parallel with the SVC. The transducer is maintained in the neutral position in the same manner as outside the body, with the probe facing anteriorly. In this view the right atrium, right ventricle and tricuspid

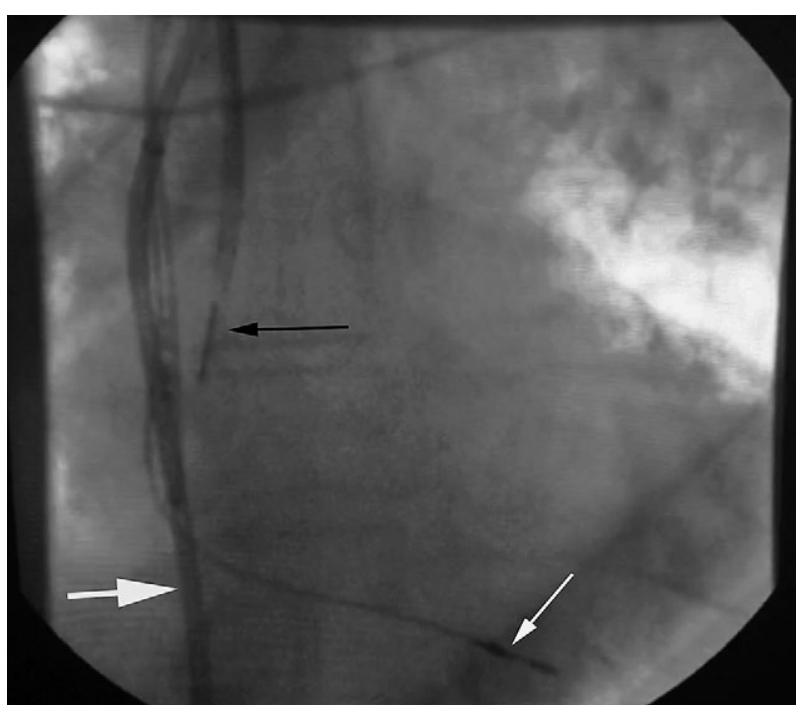

Fig 3. Superior approach. Radiological image with placement of the catheters during transseptal puncture using a superior approach. The ICE probe (black arrow) is inserted through the right subclavian vein in the middle of the right atrium. It is oriented towards the interatrial septum. The needle, dilator and sheath (large arrow) are introduced through the inferior vena cava. At the apex of the right ventricle an active fixation pacing lead can be observed (white arrow).

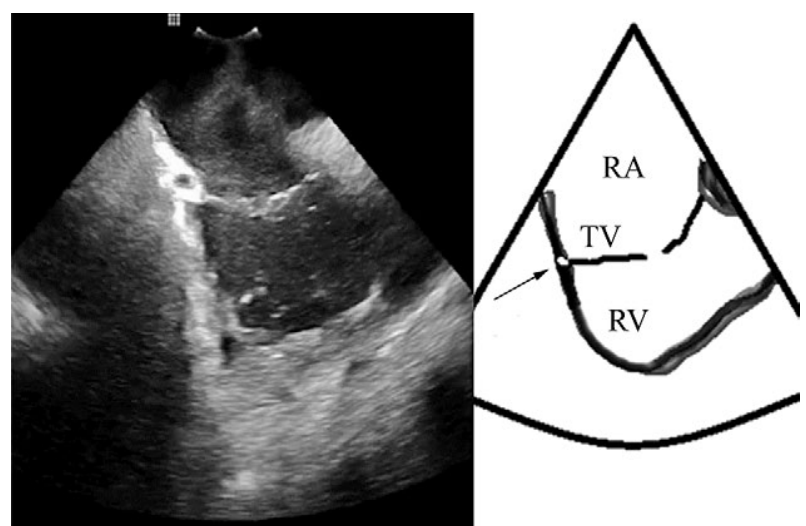

Fig 4. "Home view". Before any transseptal puncture the pericardium is verified for pericardial effusion. In the "home view" with the probe placed in the middle of the right atrium the following structures are visible: the right atrium (RA), ventricle (RV) and tricuspid valve (TV). At the level of the tricuspid valve the right coronary artery can be observed (arrow).

valve should be clearly seen (fig 4). A pericardial effusion should be excluded before any transseptal puncture.

By rotating the catheter $30^{\circ}$ clockwise, the aortic valve and the ascending aorta come into view, in close proximity to the right ventricular outflow tract (RVOT), the tricuspid valve and the pulmonary artery (fig 5, fig 6).

By performing a clockwise rotation of $60^{\circ}$ from the „home view”, the left ventricle, the mitral valve and the left atrium can be seen. In the anterior part of the left atrium, close to the mitral valve, the left atrial appendage (LAA) can be identified. If TEE was not performed previous to the transseptal puncture, ICE can be used to exclude the presence of a LAA thrombus by directly visualising the LAA and by measuring the velocity at $1 \mathrm{~cm}$ inside LAA (fig 7).

A counter clockwise rotation of $60^{\circ}$ should bring back the ,home view”.

\section{STEP 2: "Septal view"}

From the „home view” the ICE probe is flexed posteriorly and rotated clockwise $90^{\circ}$, so the transducer faces the interatrial septum. This view is comparable with the bicaval view from TEE with superior/inferior orientation of the interatrial septum (fig 8, fig 9). On a chest X-ray, the position of the catheter will correspond to the lateral wall of the right atrium. The image obtained with this position shows the interatrial septum, the right and left atria, the coronary sinus and the pulmonary veins or the LAA, depending on the exact location of the transducer, facing posterior (for the pulmonary veins) or anterior (for the LAA).

From this view, by further clockwise rotation $\left(180^{\circ}\right.$ from the "home view") the right pulmonary veins can be examined. A longitudinal view of the right pulmonary veins can be obtained by deflecting the ICE probe in a right to left plane. 


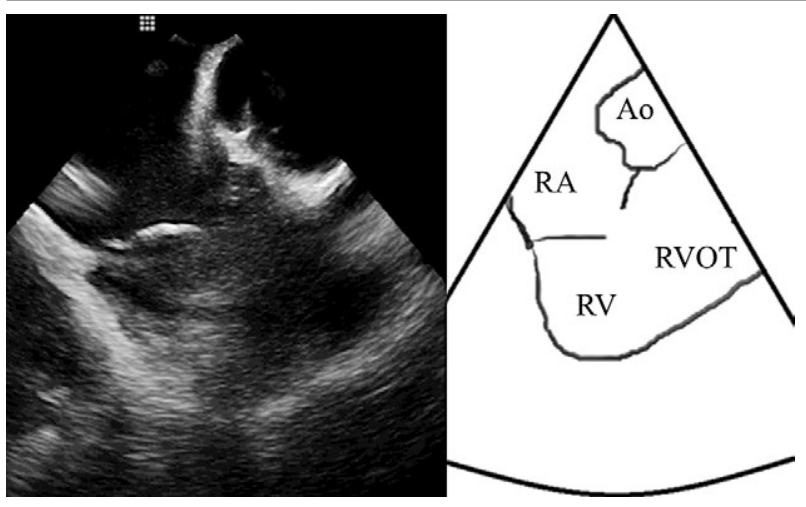

Fig 5. "Home view with aortic bulb". By making a slight $30^{\circ}$ clockwise rotation from the "home view", the aortic bulb can be visualized close to the right cavities (RA - right atrium, RV - right ventricle). The aortic bulb (Ao) is an anterior structure that should be avoided during the transseptal puncture because of the bleeding risk. RVOT - right ventricular outflow tract

By further applying a clockwise rotation, the transducer will move near the tricuspid valve annulus and inferior to the aortic valve, obtaining the ,short axis view". In this view the aorta will be displayed together with the interatrial septum offering anterior/posterior orientation for the transseptal puncture. This view corresponds to the "short-axis view" in TEE but the near-field is represented by the right atrium and not the left atrium like in TEE.

\section{STEP 3: Tenting of the fosa ovalis}

When the sheath and the dilator are positioned in the middle of the fossa the „tenting effect” occurs, because of the fibroelastic consistence of the fosa ovalis. The presence of this sign allows a precise puncture of the septum outside the muscular part of it, which can lead to pericardial effusion (fig 10).

In order to avoid complications, the fossa ovalis should be accurately visualized. The septum should be crossed in the posterior part of the fossa to avoid puncture of the aortic root. A puncture that is made anteriorly directs the needle, sheath and the catheters towards the left atrial appendage risking perforation. Furthermore, an anterior puncture makes the manipulation of electrophysiology catheters difficult in the posterior part of the left atrium and pulmonary veins. In order to guide such a posterior puncture of the fosa ovalis, the ICE catheter is rotated clockwise, to visualize the left superior pulmonary vein (LSPV) and left inferior pulmonary vein (LIPV). The superior and inferior position of the transseptal needle against the septum can be assessed by slightly advancing the ICE probe along the septum. The anterior and posterior position of the needle against the septum can be assessed by performing a clockwise (posterior) and counter clockwise (anterior) rotation. For

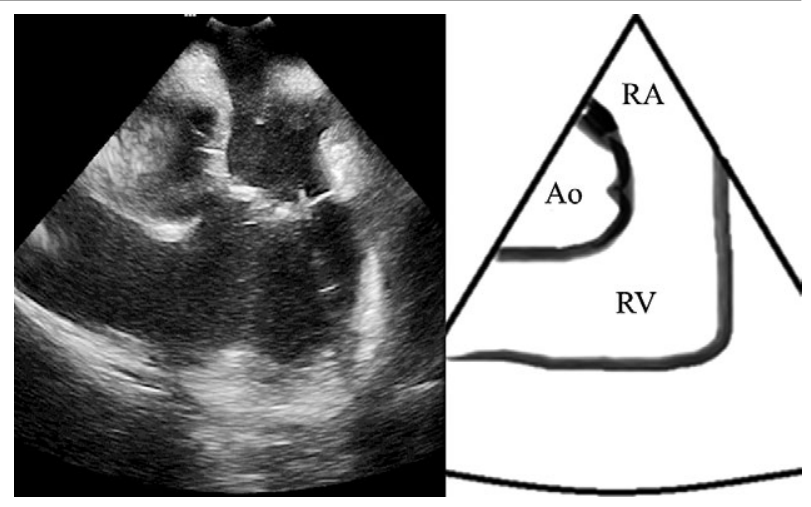

Fig 6. Superior approach to "Home view". The image shows the "home view" with the ICE probe inserted in the superior vena cava via a right subclavian vein. Compared to the same view obtained with the probe inserted from the inferior vena cava, the aorta is on the left side of the right ventricular outflow tract (RVOT). RA - right atrium, RV - right ventricle

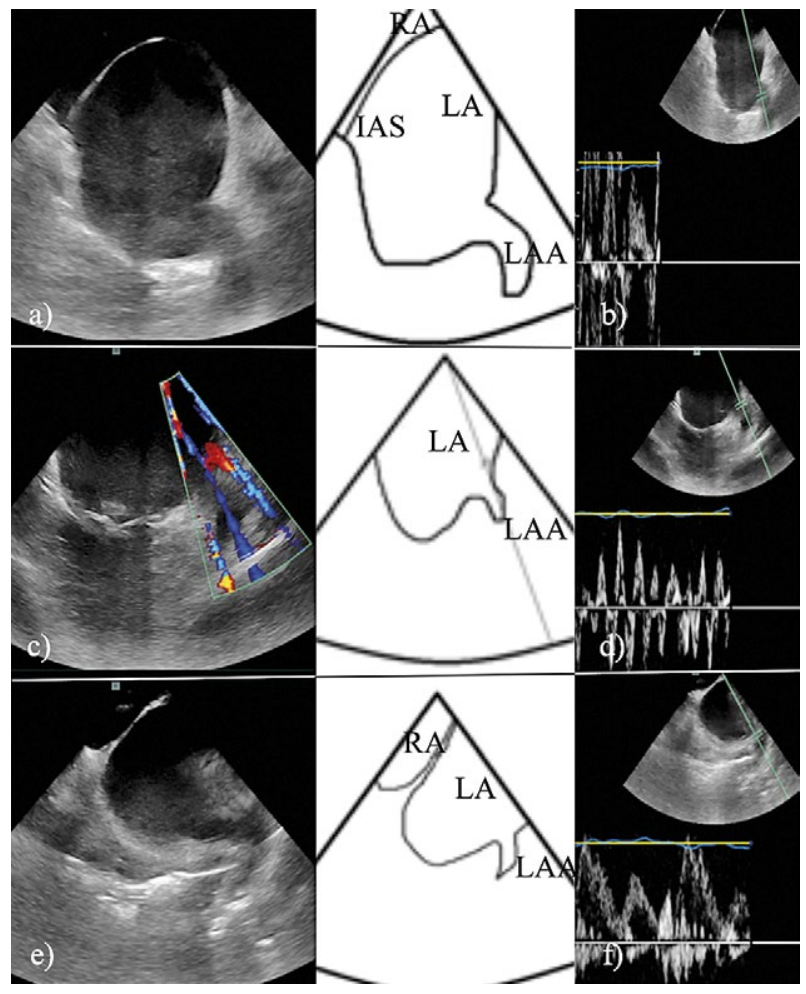

Fig 7. Left chambers. An anterior rotation from the "home view" brings the left atrial appendage (LAA) into image: a) The "septal view" shows the interatrial septum between the right atrium (RA) and left atrium (LA). The left appendage is also visible with no thrombus at this level; b) pulsed Doppler characterization of the left atrial appendage flow; c) color Doppler of the LAA; d) pulsed Doppler of the LAA shows emptying velocities of $25-30 \mathrm{~cm} / \mathrm{sec}$, the patient being in atrial fibrillation; e) interatrial septum between the right and left atria; f) pulsed Doppler of the LAA shows normal flow during sinus rhythm. 


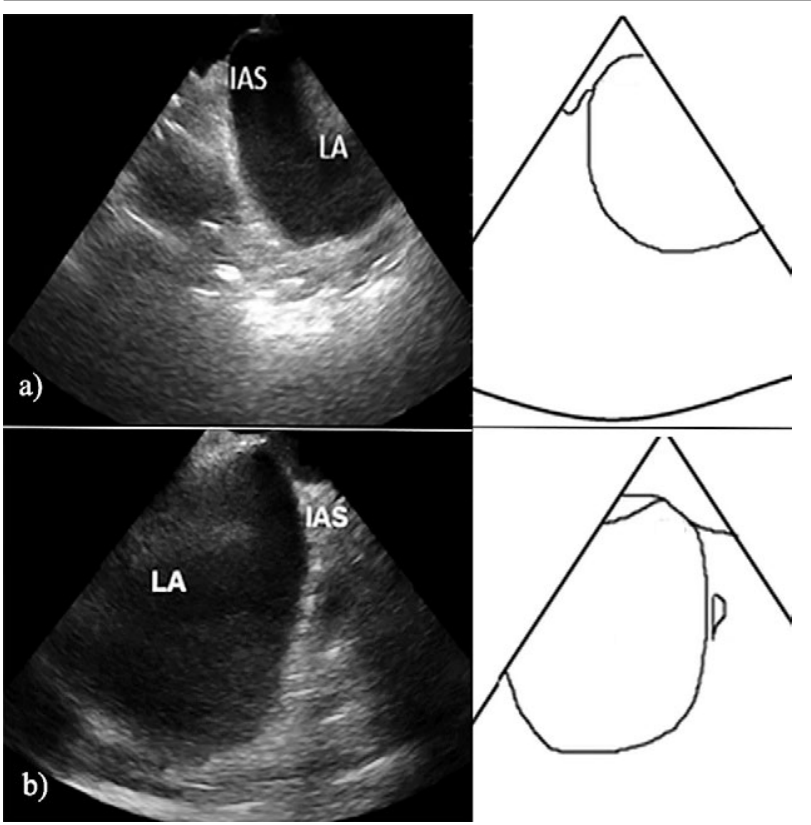

Fig 8. Septal view. Visualization of fosa ovalis from the inferior and superior approach: a) note that the septum between the right and left atrium is convex towards the right atrium, making it difficult to position the sheath and dilator on the fosa ovalis; b) interatrial septum seen from the superior vena cava in a right subclavian approach.

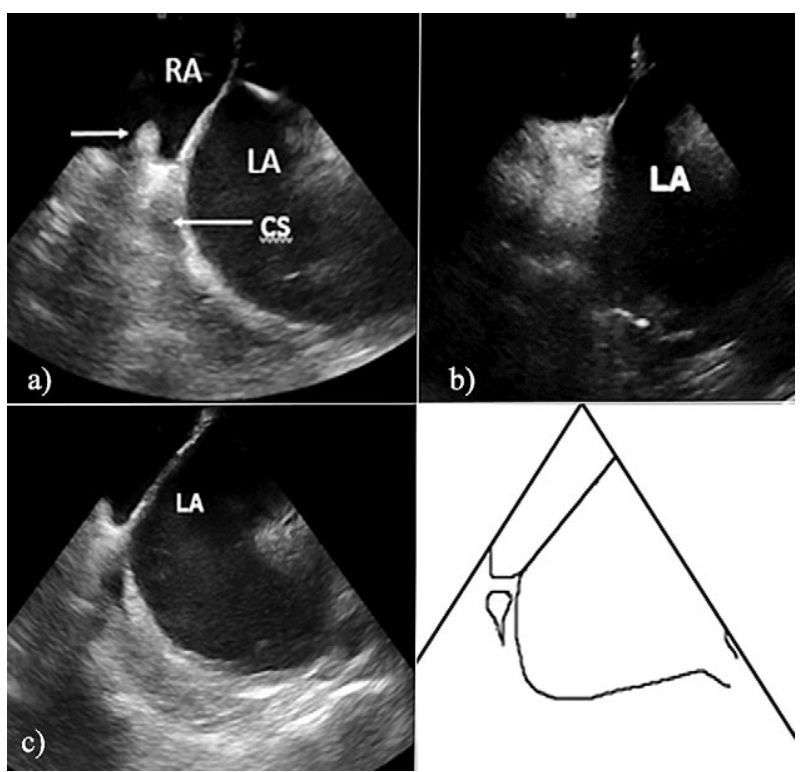

Fig 9. "Septal view". Visualization of fosa ovalis: a) Eustachian ridge (ER) and coronary sinus (CS) are also visible at the level of right atrium. Eustachian ridge is an atrial structure that separates the orifice of the inferior vena cava from the ostium of the coronary sinus and continues with the crista terminalis; b) small fosa ovalis in a patient with lipomatous septum; the puncture should be performed at the level of the fosa and not the hypertrofiated septum; c) in this patient the septum is long and thick. Coronary sinus is also visible (Cs)

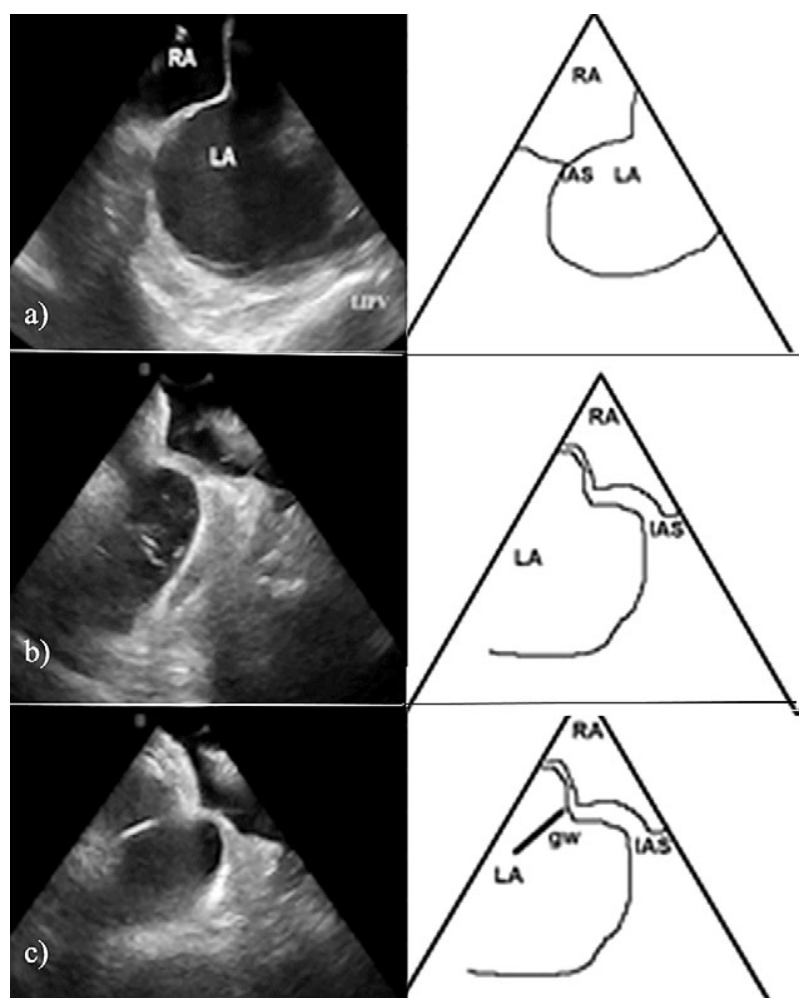

Fig 10. Brockenbrough needle against the fossa ovalis: a) typical "tenting" of the fossa ovalis, just before the puncture; the ICE probe was inserted through the inferior vena cava; b) tenting of the interatrial septum with the ICE probe inserted through the superior vena cava (please note the inverse image compared with a); c) passing of the interatrial septum with the guidewire

the aortic valve visualization an anterior alignment of the transseptal needle is required, while for the far-field pulmonary vein visualization a posterior alignment of the needle is needed.

\section{STEP 4: Crossing the interatrial septum}

After the optimal positioning of the dilator and sheath against the fossa ovalis with visualization of the tenting sign, the needle is advanced inside the left atrium. Injection of physiological serum or contrast dye inside the left atrium confirms successful puncture by the presence of microbubbles in the LA (fig 11). After a successful puncture, a guidewire is advanced into the LSPV or LIPV to stabilise the transseptal sheath and the dilator. The system is then advanced back and forth through the septum to dilate the puncture site. After the transseptal puncture is performed, anticoagulation with unfractionated heparin is carried out, in order to maintain an ACT (activated clotting time) of 2-3 times the normal value.

After the retraction of the Brockenbrough needle a second transseptal puncture is performed and the ablation catheter with the lasso catheter are advanced inside the left atrium and pulmonary veins. 


\section{STEP 5: Left atrium and pulmonary veins}

Once the transseptal puncture is made, ICE can give important information on the number, position and size of

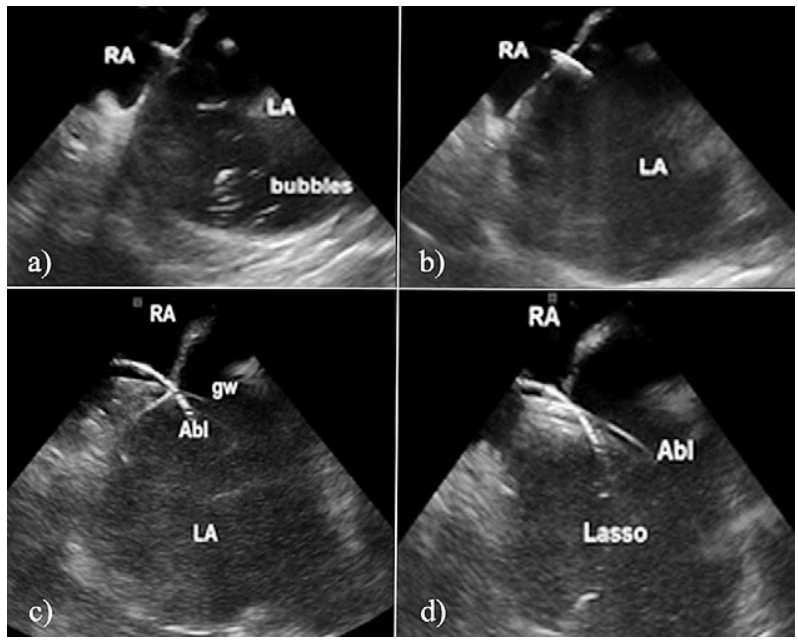

Fig 11. Successful transseptal puncture: a) bubbles in the left atrium confirm the successful transseptal puncture. Serum or contrast dye can be injected in the left atrium as they both show bubbles; b) transseptal sheath passes the septum and goes inside the left atrium; c) a second guidewire (gw) is inserted through the interatrial septum, beside the ablation catheter; d) using the second guidewire another transseptal sheath is introduced in the left atrium together with a Lasso catheter.

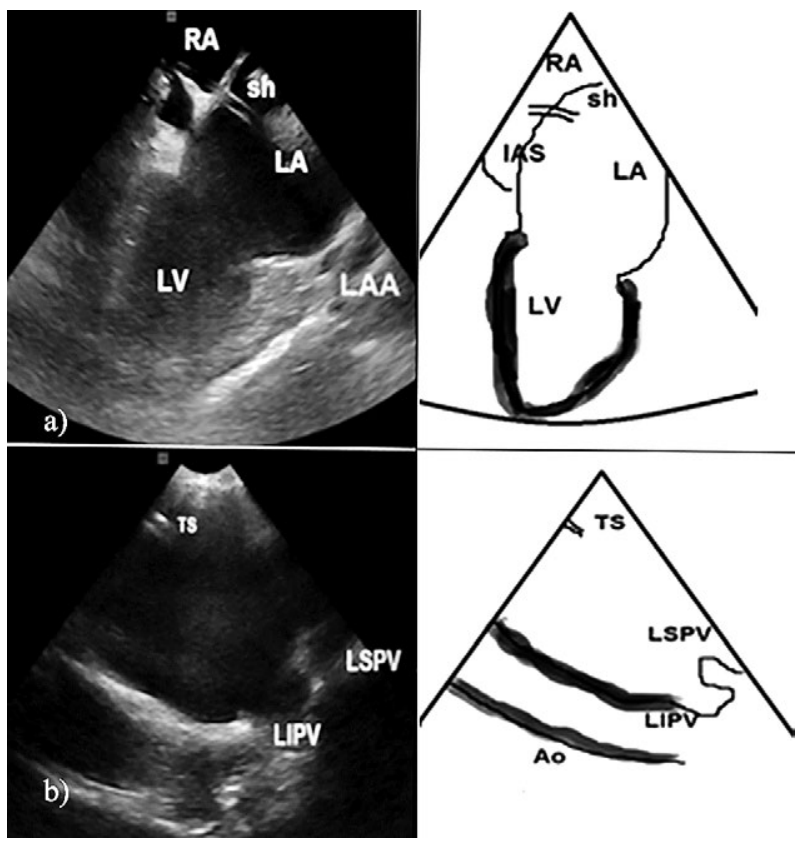

Fig 12. Anterior and posterior puncture of the fossa ovalis: a) transseptal puncture was performed in the anterior part of the fosa ovalis; therefore, the left ventricle and LAA are visible b) transseptal puncture was performed in the posterior part of the fosa ovalis; therefore, the left superior and inferior pulmonary veins are visible. the pulmonary veins, as well as the presence of a common trunk between the 2 left veins. The LAA can be assessed in terms of size, form, anterior position and spatial relationship with the pulmonary veins (fig 12). It will also offer real-time information on the precise location of the ablation catheter and lasso catheter inside the left atrium or pulmonary veins (fig 13-16). Contact with the antrum of the pulmonary veins can also be assessed, as well as the exact position of the lasso catheter inside the pulmonary vein.

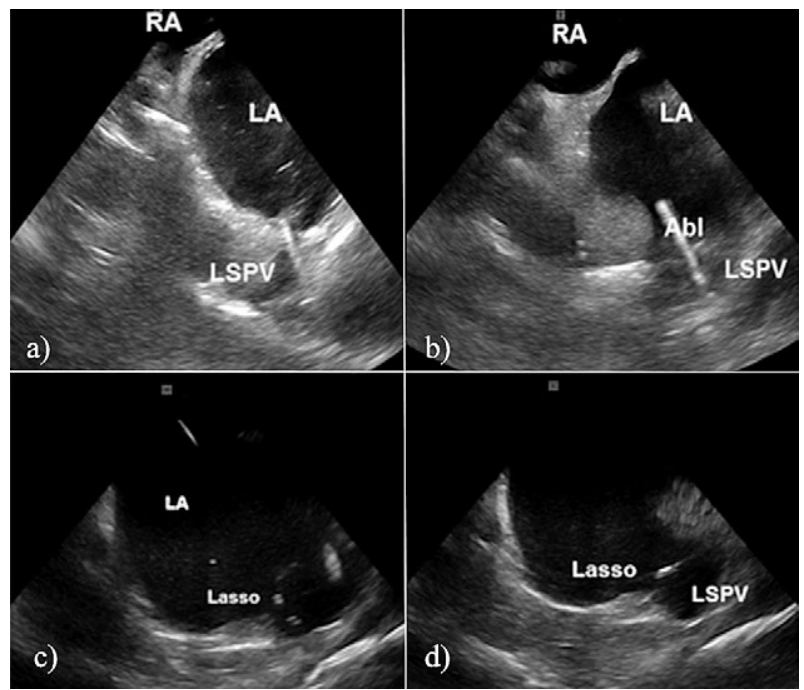

Fig 13. Ablation and Lasso catheter inside the left superior pulmonary vein: a) the ablation catheter is deep inside the LSPV towards the pleura of the left lung; b) the ablation catheter is retraced towards the ostium of the LSPV; c) lasso catheter inside the LSPV.

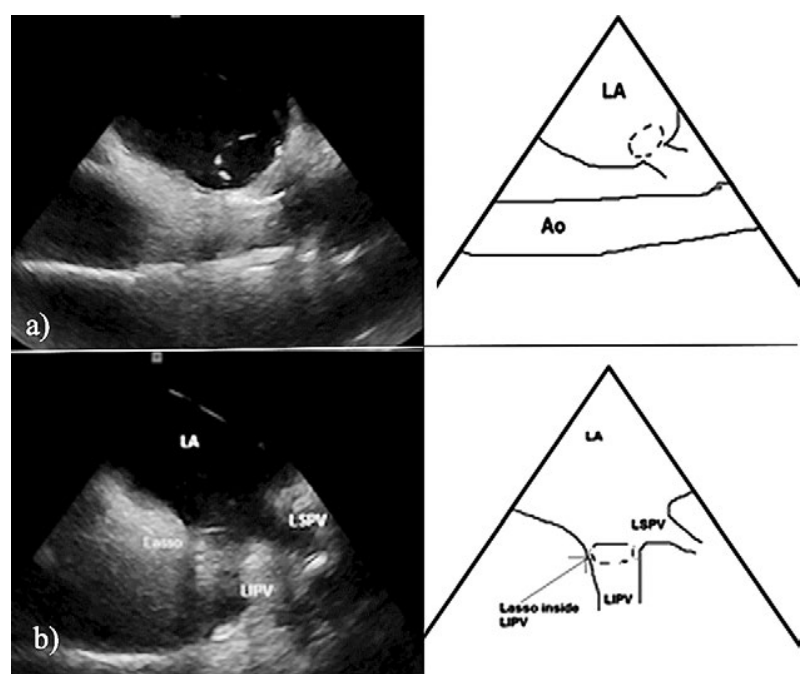

Fig 14. Lasso catheter inside the left inferior pulmonary vein: a) lasso catheter at the level of the left inferior pulmonary vein; b) both left superior and inferior pulmonary veins are visible; the Lasso catheter is inserted in the LIPV. 


\section{STEP 6: Left ventricular long-axis view}

At the end of the procedure, the catheters and the sheaths are retracted inside the right atrium, leaving a small atrial septal defect. Because of the small size of the catheters, the residual defect is small and clinically in-

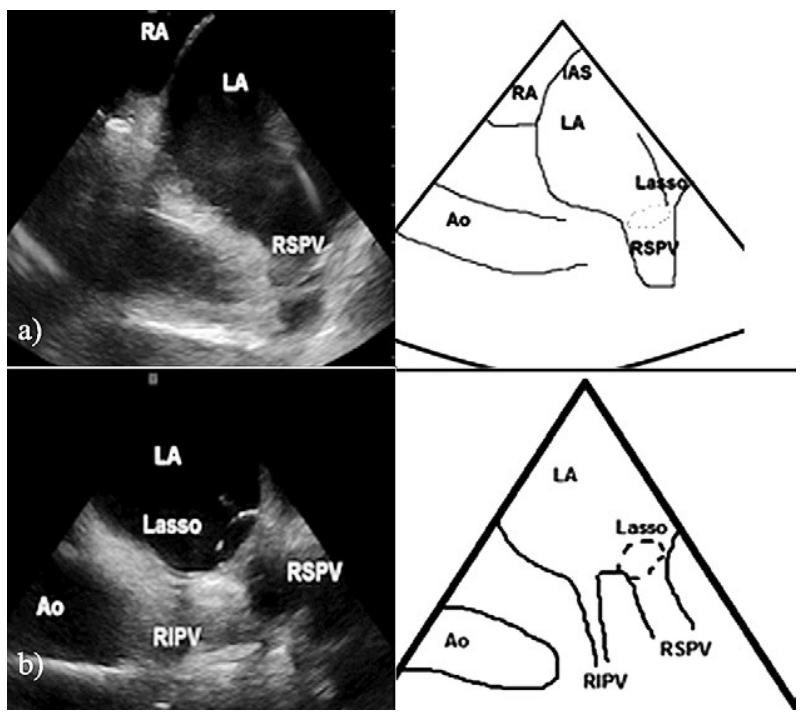

Fig 15. Lasso catheter at the level of the right superior pulmonary vein: a) from the "septal view", a clockwise rotation brings into image the right superior pulmonary vein (RSPV). The vein is distal to the ICE probe; therefore, it is situated in the bottom of the image, draining in the body of the right atrium; b) A further rotation of the probe will display superior and inferior right pulmonary veins. A Lasso catheter is inserted at the ostium of the RSPV.

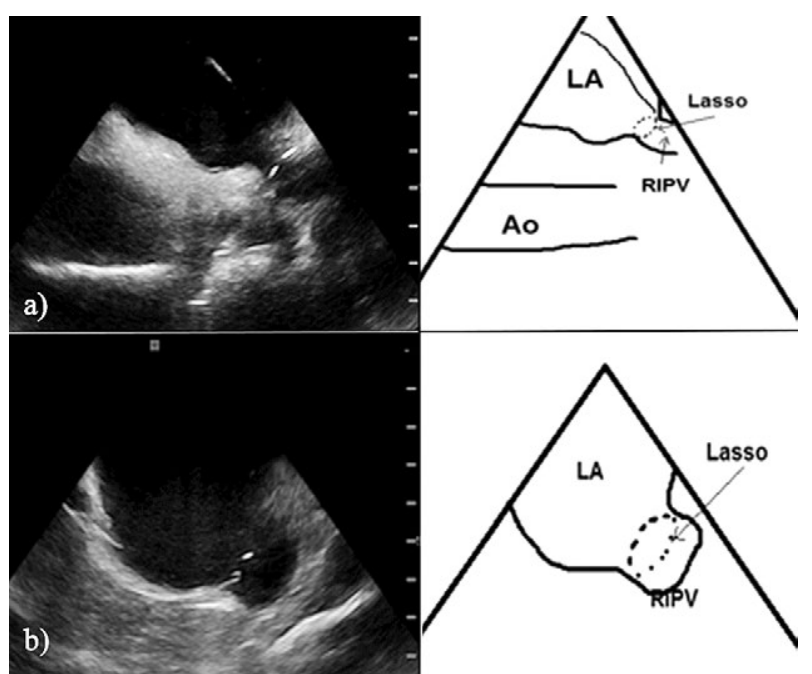

Fig 16. Lasso catheter at the level of the right inferior pulmonary vein: a) from the septal view a further clockwise rotation will bring the RIPV into image. RIPV is more posterior compared to RSPV which is an anterior vein. A Lasso catheter is inserted at the level of the RIPV ostium.

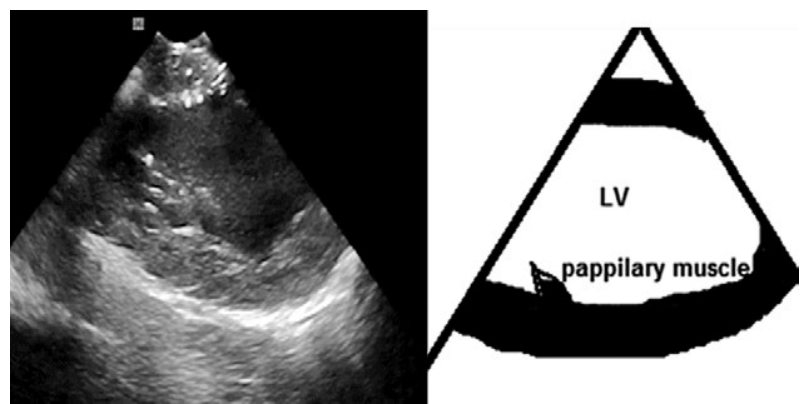

Fig 17. Left ventricular view. There is no pericardial effusion at the end of procedure.

significant. It usually closes completely within 3 months [19]. Before the ICE catheter is completely removed, it should be flexed anteriorly and rotated to pass from the right atrium through the tricuspid valve inside the right ventricle. From this position, the left ventricle can be visualized and a pericardial effusion can be excluded at the end of the ablation procedure (fig 17).

\section{Complications of transseptal puncture}

In patients with abnormal anatomy the transseptal puncture is difficult because of the atypical position of the interatrial septum. Radiological guidance without ICE does not permit a good localization of the septum and complications can occur: pericardial tamponade, aortic root puncture, arterial embolism with atrial thrombus, perforation of pulmonary veins or of the inferior vena cava [20]. ICE-guided puncture was developed to avoid life threatening complications and to increase the success rate of an effective puncture in the case of an abnormal septum location.

\section{Conclusion}

ICE is used in the electrophysiology laboratory to increase the safety of the transseptal puncture and to guide the catheter ablation procedures. It allows for early detection of procedure-related complications such as pericardial effusion, aortic root puncture or catheter thrombosis. It can be used by the cardiac electrophysiologist without the presence of a dedicated sonographer in the EP room. Importantly, it significantly reduces contrast dye administration and X-ray radiation exposure and may avoid general anaesthesia. For all these reasons, ICE is a valuable tool that should be part of any modern electrophysiology laboratory or catheterization lab dedicated to structural heart disease interventions.

\section{Conflict of interest: none}




\section{References}

1. Basman C, Parmar YJ, Kronzon I. Intracardiac echocardiography for structural heart and interventional electrophysiological interventions. Curr Cardiol Rep 2017:19:102.

2. Ren JF, Marchlinski FE. Utility of intracardiac echocardiography in left heart ablation for tachyarrhythmias. Echocardiography 2007;24:533-540.

3. Marmagkiolis K, Cilingiroglu M. Intracardiac echocardiography-guided percutaneous mitral baloon valvuloplasty. Rev Port Cardiol 2013;32:337-339.

4. Cismaru G, Muresan L, Rosu R, et al. Intracardiac echocardiography to guide catheter ablation of an accesory pathway in Ebstein's anomaly. A case report. Med Ultrason 2018;20:250-253.

5. Berti S, Pastormelo LE, Celi S, et al. First-in-human percutaneous left atrial appendage occlusion procedure guided by real-time-3 dimensional intracardiac echocardiography. JACC Cardiovasc Intervent 2018;11:2228-2231.

6. Kavvouras C, Vavuranakis M, Vaina S, et al. Intracardiac echocardiography for percutaneous patent foramen ovale and atrial septal deffect occlusion. Herz 2018 Jan 26. doi:10.1007/s00059-017-4678-7.

7. Alqahtani F, Bhirud A, Aljohani S, et al. Intracardiac versus transesophageal echocardiography to guide transcatheter closure of interatrial communications: nationwide trend and comparative analysis. J Interv Cardiol 2017;30:234-241.

8. Caldararu C, Balanescu S. Modern use of echocardiography in transcatheter aortic valve replacement: an Up-Date. Maedica (Buchar). 2016;11:299-307.

9. Bouajila S, Chatard A, Dauphin C. Usefulness of intracardiac echocardiography for the diagnosis of infective endocarditis following percutaneous pulmonary valve replacement. Cardiol Young 2017;27:1406-1409.

10. Homorodean C, Olinic M, Olinic D. Development of a methodology for structured reporting of information in echocardiography. Med Ultrason 2012;14:29-33.
11. Biermann J, Bode C, Asbach S. Intracardiac echocardiography during catheter-based ablation of atrial fibrillation. Cardiol Res Pract 2012;2012:921746.

12. Poanta L, Albu A, Fodor D. Chiari network-case report and brief literature review. Med Ultrason 2010;12:71-72.

13. Szili-Torok T, McFadden EP, Jordaens LJ, Roelandt JR. Visualization of elusive structures using intracardiac echocardiography: Insights from electrophysiology. Cardiovasc Ultrasound 2004;2:6.

14. Enriquez A, Tapias C, Rodriguez D, et al. Role of intracardiac echocardiography for guiding ablation of tricuspid valve arrhythmias. Heart Rhythm Case Rep 2018;4:209213.

15. Radinovic A, Mazzone P, Landoni G, Agricola E, Regazzoli D, Della Bella P. Different transseptal puncture for different procedures: optimization of left atrial catheterization guided by transesophageal echocardiography. Ann Card Anaesth 2016;19:589-593.

16. O'Brien B, Zafar H, De Freitas S, Sharif F. Transseptal puncture-Review of anatomy, techniques, compications and challenges. Int J Cardiol 2017;233:12-22.

17. Cismaru G, Schiau S, Muresan L, et al. Intravascular pulmonary venous ultrasound imaging for catheter ablation of atrial fibrillation. Expert Rev Med Devices 2017;14:309314.

18. Jensen B, Spicer DE, Sheppard MN, Anderson RH. Development of the atrial septum in relation to postnatal anatomy and interatrial communications. Heart 2017;103:456462.

19. Singh SM, Douglas PM, Reddy VY. The incidence and long-term clinical outcomeof iatrogenic atrial septal defects secondary to transseptal catheterization with a $12 \mathrm{~F}$ transseptal sheath. Circ Arrhythm Electrophysiol 2011;4:166171.

20. Salghetti F, Sieira J, Chierchia GB, Curnis A, de Asmundis C. Recognizing and reacting to complications of transseptal puncture. Expert Rev Cardiovasc Ther 2017;15:905-912. 